\title{
Changes in the interstitial cells of Cajal in the gallbladder of guinea pigs fed a lithogenic diet
}

\author{
LEI HUANG $^{1 *}$, CHAO DING $^{2 *}$ and XINMIN SI ${ }^{2}$ \\ ${ }^{1}$ Department of General Surgery, Children's Hospital of Nanjing Medical University, Nanjing, \\ Jiangsu 210008; ${ }^{2}$ Department of Gastroenterology, The First Affiliated Hospital of \\ Nanjing Medical University, Nanjing, Jiangsu 210029, P.R. China
}

Received June 11, 2020; Accepted March 12, 2021

DOI: $10.3892 / \mathrm{etm} .2021 .10255$

\begin{abstract}
Cholesterol cholelithiasis is a common disease and gallbladder hypomotility may underlie its pathogenesis. Interstitial cells of Cajal (ICCs) in the gallbladder serve vital roles in regulating gallbladder motility. The aim of the present study was to investigate changes in gallbladder ICCs during the development of cholesterol cholelithiasis. A total of 40 male guinea pigs were randomly assigned to four groups and fed a standard diet (SD) or lithogenic diet (LD) for 2 or 8 weeks. The LD significantly increased the total cholesterol levels in the serum and bile, as well as the serum levels of high-density lipoprotein-cholesterol and low-density lipoprotein-cholesterol after 2 and 8 weeks. The LD also significantly increased and decreased the number of gallbladder ICCs at 2 and 8 weeks, respectively, by regulating the stem cell factor/C-kit pathway. Moreover, the ultrastructure of gallbladder ICCs was significantly altered after 8 weeks, and the protein expression levels of connexin 43 in the gallbladder were differentially altered after 2 and 8 weeks. Finally, cholecystokinin receptor type A (CCK1R) expression in the gallbladder was assessed. In gallbladder ICCs, its expression was significantly increased and decreased after 2 and 8 weeks, respectively. In conclusion, these results demonstrate that the density, ultrastructure and CCK1R expression levels of gallbladder ICCs are differentially altered at various stages of cholesterol cholelithiasis progression, indicating that gallbladder ICCs may be considered a potential therapeutic target for treatment of cholesterol cholelithiasis.
\end{abstract}

Correspondence to: Professor Xinmin Si, Department of Gastroenterology, The First Affiliated Hospital of Nanjing Medical University, 300 Guangzhou Road, Nanjing, Jiangsu 210029, P.R. China

E-mail: sixinminhuanglei@163.com

${ }^{*}$ Contributed equally

Key words: cholesterol cholelithiasis, gallbladder, interstitial cells of Cajal, lithogenic diet, cholecystokinin-1 receptor

\section{Introduction}

Cholelithiasis is a highly prevalent gastroenterological disease and one of the leading causes of hospital admissions worldwide (1). In China, the incidence of cholelithiasis is $8-10 \%$, and the incidence rate has been gradually increasing in recent years (2). It was reported that females of childbearing age are approximately twice more likely than males to develop cholelithiasis (3). Furthermore, among the subtypes of cholelithiasis, cholecystolithiasis accounts for $\sim 85 \%$ of cases (4). Cholelithiasis is composed of $>90 \%$ cholesterol, and $<10 \%$ is composed of black and brown pigments, which are presented in the gallbladder or infected bile ducts (5). In recent years, gallbladder hypomotility was also found to promote cholesterol crystallization and gallstone formation (6). There is an urgent need to further clarify the possible mechanisms underlying gallbladder hypomotility in the process of cholesterol gallstone formation.

Interstitial cells of Cajal (ICCs) were first identified in 1889 (7) and have been shown to possess the capacity to generate and propagate electrical slow waves in the digestive tract (8). To date, ICCs have been detected in various tissues including the pancreas, heart, urinary tract and blood vessels (7). ICCs were also found to be distributed in the gallbladder wall where they are involved in modulating the excitability and motility of gallbladder smooth muscle (9). It was reported that the density and location of ICCs in the gallbladder of patients with cholelithiasis are notably altered $(10,11)$. Moreover, a previous study reported that the ultrastructure of gallbladder ICCs was not significantly altered in mouse models of cholesterol cholelithiasis (12). The aim of the present study was to elucidate the changes in the density and ultrastructure of gallbladder ICCs during the development of cholesterol cholelithiasis in more detail compared with the existing literature.

Cholecystokinin (CCK) is a classical type of intestinal hormone that can trigger the contraction of gallbladder smooth muscle (13). CCK receptors belong to the G protein-coupled receptor superfamily and have been classified as cholecystokinin receptor type A (CCK1R) and gastrin/cholecystokinin type B receptor (14). It has been shown that CCK1R (also termed CCK-AR) is expressed in gallbladder ICCs and serves a key role in mediating $\mathrm{CCK}$-induced evoked gallbladder 
contraction (15). However, to the best of our knowledge, no studies have shown whether CCK1R expression in the gallbladder, particularly in gallbladder ICCs, is altered during the development of cholesterol cholelithiasis.

The lithogenic diet (LD), which contains a higher proportion of cholesterol, is widely used to induce cholesterol cholelithiasis in animals $(16,17)$. The aim of the present study was to detect the changes in the density, ultrastructure and CCK1R expression levels in gallbladder ICCs in LD-fed guinea pigs.

\section{Materials and methods}

Animal models. A total of 40 male guinea pigs (4 weeks old; weight, 250-280 g) were purchased from Charles River Laboratories, Inc. and randomly assigned to four groups $(n=10)$ : Standard diet (SD) for 2 weeks; SD for 8 weeks; LD for 2 weeks and LD for 8 weeks. Guinea pigs in the LD group were fed with LD (chow diet supplemented with $\sim 1.25 \%$ cholesterol, $0.5 \%$ bile salt and $15 \%$ fat) and animals in the SD group were fed standard chow. All guinea pigs were maintained in cages with a 12-h light/dark cycle with free access to food and water. All animal experiments were performed according to the National Institutes of Health Guide for the Care and Use of Laboratory Animals and approved by the Institutional Animal Care and Use Committee of the First Affiliated Hospital of Nanjing Medical University (Nanjing, China).

Measurement of total cholesterol (T-CHOL), lipoproteins and CCK octapeptide (CCK8) levels in the serum and bile. After feeding for 2 or 8 weeks, guinea pigs in the SD group (weighing 340-800 g) and the LD group (weighing 420-1200 g) were anesthetized by 1.5-3.0\% isoflurane, and blood samples were obtained from the eyes of guinea pigs $(\sim 1 \mathrm{ml}$ per guinea pig) and collected in tubes containing heparin. Plasma was obtained following centrifugation at $1,789 \mathrm{x} g$ for $15 \mathrm{~min}$ at $4^{\circ} \mathrm{C}$. Moreover, after resecting the gallbladder, bile was collected and diluted with saline water (1:10), followed by centrifugation at $2,795 \mathrm{xg}$ for $10 \mathrm{~min}$ at $4^{\circ} \mathrm{C}$ to remove the granular components. Following which, all guinea pigs were euthanized by an overdose of isoflurane. To degrade the bile pigment, bile was illuminated under a daylight lamp for $12 \mathrm{~h}$ at $4^{\circ} \mathrm{C}$. The serum levels of T-CHOL, high-density lipoprotein cholesterol (HDL-C) and low-density lipoprotein cholesterol (LDL-C), as well as the T-CHOL levels in bile were measured using an automatic biochemical analyser (Hitachi, Ltd.) with the corresponding reagents (T-CHOL, cat. no. SNM226; HDL-C, cat. no. SNM224; LDL-C, cat. no. SNM222; all purchased from Beijing Biolab Science $\&$ Technology Co., Ltd.). The concentration of CCK8 in serum was measured using an ELISA kit (cat. no. abx150409; Abbexa Ltd.) according to the manufacturer's protocol.

Immunofluorescence staining. After snap-freezing, the resected gallbladders were cut into $5-\mu \mathrm{m}$ sections using a freezing microtome. Subsequently, all sections were fixed in $4 \%$ paraformaldehyde for $20 \mathrm{~min}$ at room temperature and washed three times with PBS. To prevent non-specific binding, sections were incubated with immunostaining blocking buffer (Beyotime Institute of Biotechnology) for $1 \mathrm{~h}$ at room temperature. Subsequently, sections were incubated overnight at $4{ }^{\circ} \mathrm{C}$ with the following primary antibodies: Anti-C-kit (1:200; cat. no. ab25022; Abcam) and anti-CCK1R (5 $\mu \mathrm{g} / \mathrm{ml}$; cat. no. ab77269; Abcam). Following three washes with PBS, sections were incubated with rabbit anti-rat IgM/Alexa Fluor 488 antibody (1:500; cat. no. bs-0346R; BIOSS) and rabbit anti-goat IgG/Alexa Fluor 647 antibody (1:500; cat. no. bs-0294R; BIOSS) for $2 \mathrm{~h}$ at room temperature, and then incubated with DAPI (Beyotime Institute of Biotechnology) for $10 \mathrm{~min}$ at room temperature. Finally, sections were mounted using antifade mounting medium (Beyotime Institute of Biotechnology). For the negative control, the primary antibodies were omitted. Images were captured on a laser scanning confocal microscope (magnification, x200 or x400). C-kit-positive cells excluding those with a round shape (mast cells) were considered as gallbladder ICCs. To quantify the number of gallbladder ICCs, three gallbladders were obtained from each group, three sections were prepared from each gallbladder and three random fields of view (magnification, $\mathrm{x} 200$ ) in each section were selected. The number of gallbladder ICCs in the lamina propria was counted by two researchers in a blinded manner.

Western blot analysis. The resected gallbladders were washed with PBS and lysed in RIPA lysis buffer (Thermo Fisher Scientific, Inc.) to extract total protein. Protein concentration was measured using a BCA protein assay kit (Beyotime Institute of Biotechnology) according to the manufacturer's protocol. Subsequently, $50 \mu \mathrm{g}$ of protein was loaded on an $8 \%$ SDS gel, resolved using SDS-PAGE and subsequently transferred to a PVDF membrane (EMD Millipore). PVDF membranes were blocked in TBS (Boster Biological Technology) containing $5 \%$ skimmed milk powder for $2 \mathrm{~h}$ at room temperature. Subsequently, membranes were washed three times with TBS supplemented with $0.05 \%$ Tween-20 and incubated overnight at $4{ }^{\circ} \mathrm{C}$ with one of the following antibodies: Anti-C-kit (1:1,000; cat. no. ab256345; Abcam), anti-stem cell factor (SCF; 1:1,000; cat. no. ab64677; Abcam), anti-Connexin (Cx) 43 (1:2,000; cat. no. ab11370; Abcam), anti-CCK1R (1:500; cat. no. ab75153; Abcam) or anti-GAPDH $(1: 1,000$; cat. no. AF0006; Beyotime Institute of Biotechnology), followed by incubation with horseradish peroxidaseconjugated goat anti-rabbit IgG (1:5,000; cat. no. bs-0295G; BIOSS) for $2 \mathrm{~h}$ at room temperature. Protein signals were detected using an enhanced chemiluminescence detection kit (Thermo Fisher Scientific Inc.) on the ChemiDoc XRS+ chemiluminescence imaging system (Bio-Rad Laboratories. Inc.). Densitometric analysis was performed using Image J v1.8.0 software (National Institutes of Health), and expression of each protein was normalized to the respective GAPDH loading control band.

Transmission electron microscopy. Gallbladder specimens were fixed in $2.5 \%$ glutaraldehyde/ $0.1 \mathrm{M}$ cacodylate buffer for $2 \mathrm{~h}$ at $4^{\circ} \mathrm{C}$ and $1 \%$ perosmic acid for $2 \mathrm{~h}$ at room temperature. Specimens were then dehydrated using a series of ethanol solutions of increasing concentration and embedded in Epon ethoxyline resin at $60^{\circ} \mathrm{C}$. Ultrathin sections with 70-80-nm thickness were obtained using a Leica EM UC7 ultramicrotome (Leica Microsystems, Inc.) and subsequently placed on 
A

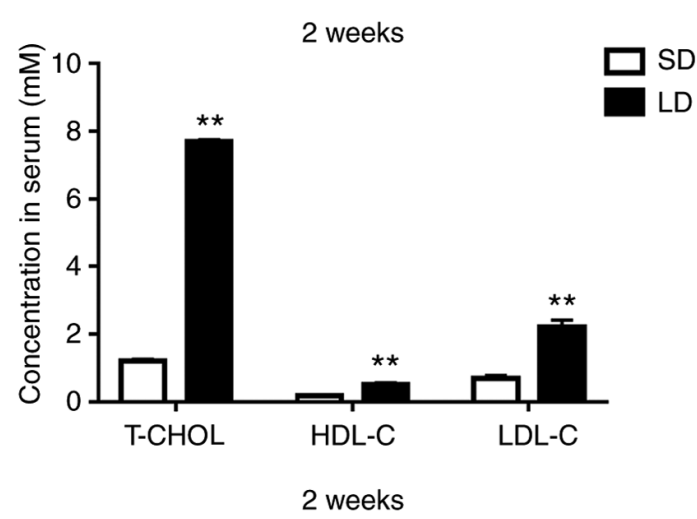

C

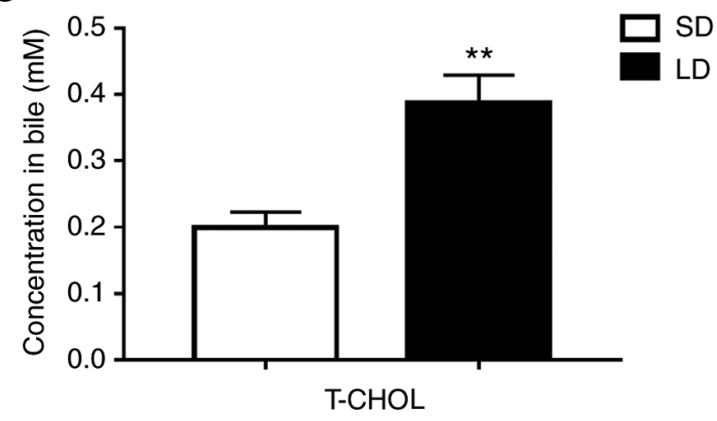

B
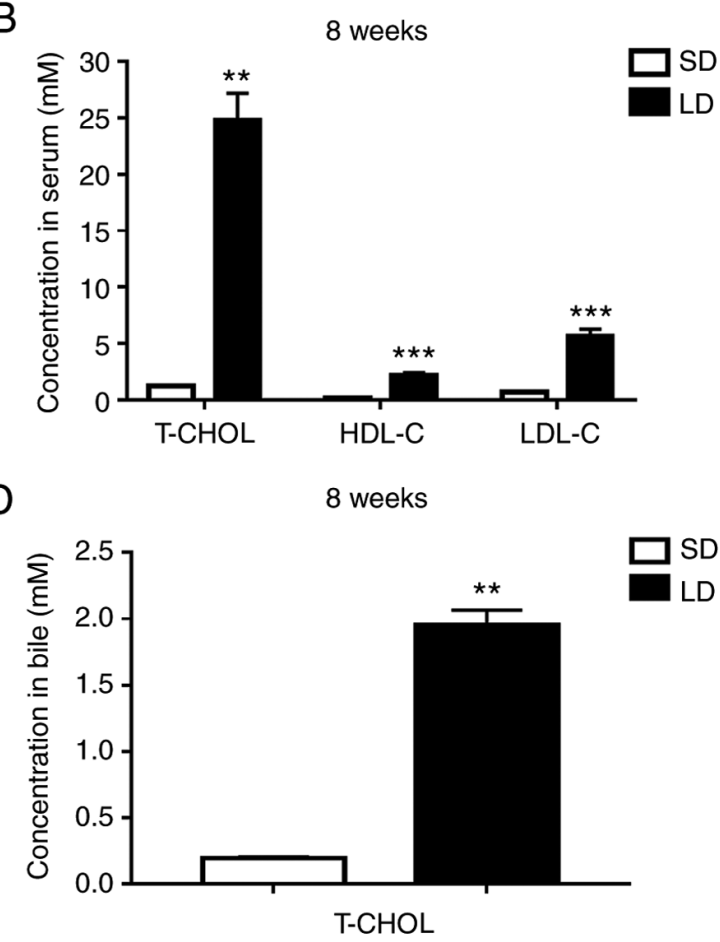

Figure 1. LD significantly increases T-CHOL levels in the serum and bile and the serum levels of HDL-C and LDL-C. Serum levels of T-CHOL, HDL-C and LDL-C were measured after (A) 2 and (B) 8 weeks in guinea pigs fed with SD or LD. The bile levels of T-CHOL were measured after (C) 2 and (D) 8 weeks in guinea pigs fed with SD or LD. ${ }^{* *} \mathrm{P}<0.01$ and ${ }^{* * *} \mathrm{P}<0.001$ vs. SD/2 weeks or SD/8 weeks. SD, standard diet; LD, lithogenic diet; HDL-C, high-density lipoprotein cholesterol; LDL-C, low-density lipoprotein cholesterol; T-CHOL, total cholesterol.

grids prior to staining with $1 \%$ uranyl acetate for $20 \mathrm{~min}$ at room temperature. Sections were examined under a transmission electron microscope (H-7500; Hitachi, Ltd.) at a magnification of $x 10,000$ at $80 \mathrm{kV}$.

Statistical analysis. Data were presented as the mean \pm standard deviation. Significant differences between the SD group and LD group were analysed using SPSS version 22.0 (IBM Corp.) using an unpaired two-tailed Student's t-test. $\mathrm{P}<0.05$ was considered to indicate a statistically significant difference.

\section{Results}

LD significantly increases $T$-CHOL levels in the serum and bile and serum levels of $H D L-C$ and $L D L-C$. The serum levels of T-CHOL, HDL-C and LDL-C were significantly increased in guinea pigs fed a LD for 2 weeks compared with those fed a SD for the same time period (SD/2 weeks vs. LD/2 weeks: T-CHOL, 1.210 $\pm 0.051 \mathrm{mM}$ vs. 7.698 $\pm 0.046 \mathrm{mM}$; HDL-C, $0.179 \pm 0.035 \mathrm{mM}$ vs. $0.513 \pm 0.061 \mathrm{mM}$; LDL-C, $0.693 \pm 0.093 \mathrm{mM}$ vs. 2.211 $0.210 \mathrm{mM}$; Fig. 1A). Moreover, more significant increases in the serum levels of T-CHOL, HDL-C and LDL-C were observed in guinea pigs fed a LD for 8 weeks (SD/8 weeks vs. LD/8 weeks: T-CHOL, $1.228 \pm 0.065 \mathrm{mM}$ vs. $24.729 \pm 2.461 \mathrm{mM}$; HDL-C, $0.178 \pm 0.011 \mathrm{mM}$ vs. $2.175 \pm 0.272 \mathrm{mM}$; LDL-C, $0.699 \pm 0.070 \mathrm{mM}$ vs. 5.631 $\pm 0.651 \mathrm{mM}$; Fig. 1B). Furthermore, the bile levels of T-CHOL in guinea pigs fed a LD for 2 weeks was significantly higher compared with those fed a SD for 2 weeks (SD/2 weeks vs. LD/2 weeks: 0.199 $\pm 0.023 \mathrm{mM}$ vs. $0.388 \pm 0.042 \mathrm{mM}$; Fig. 1C). Similarly, the bile levels of T-CHOL in guinea pigs fed a LD for 8 weeks were also significantly increased compared with those fed a SD for 8 weeks (SD/8 weeks vs. LD/8 weeks: $0.198 \pm 0.010 \mathrm{mM}$ vs. $1.955 \pm 0.112 \mathrm{mM}$; Fig. 1D).

LD significantly alters the number of ICCs and the protein expression levels of $C$-kit and SCF in guinea pig gallbladders. As shown in Fig. 2A, ICCs in guinea pig gallbladders were labelled using a primary antibody targeting C-kit (a marker of ICCs, indicated by green fluorescence in Fig. 2A). The mean number of ICCs in the lamina propria of guinea pig gallbladders at x200 magnification was significantly increased in the $\mathrm{LD} / 2$ weeks group compared with the SD/2 weeks group (SD/2 weeks vs. LD/2 weeks: $19.4 \pm 3.1$ vs. $24.1 \pm 3.2$ ), and was significantly decreased in the $\mathrm{LD} / 8$ weeks group compared with the $\mathrm{SD} / 8$ weeks group (SD/8 weeks vs. LD/8 weeks: $19.9 \pm 3.2$ vs. $10.9 \pm 2.1$; Fig. 2B). Moreover, western blot analysis demonstrated that the protein expression levels of $\mathrm{C}$-kit and SCF in guinea pig gallbladders were significantly increased in the $\mathrm{LD} / 2$ weeks group compared with the SD/2 weeks group (C-kit, 20\%; SCF, 40\%), and were significantly reduced in the $\mathrm{LD} / 8$ weeks group compared with the $\mathrm{SD} / 8$ weeks group (C-kit, 58\%; SCF, 62\%; Fig. 2C and D).

LD significantly alters the ultrastructure of gallbladder ICCs in guinea pigs fed an LD for 8 weeks and the protein expression levels of Cx43 in guinea pig gallbladders. Next, the ultrastructure of gallbladder ICCs in guinea pigs fed a LD was assessed using transmission electron microscopy. The results showed that gallbladder ICCs in the guinea pigs fed a SD possessed 
A
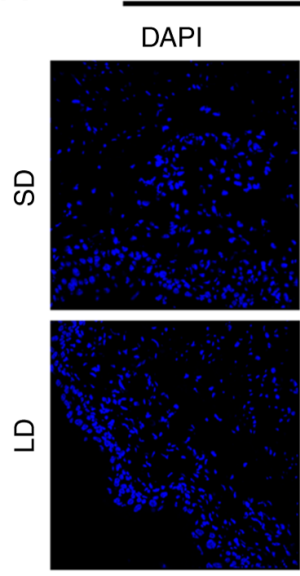

$\mathrm{B}$

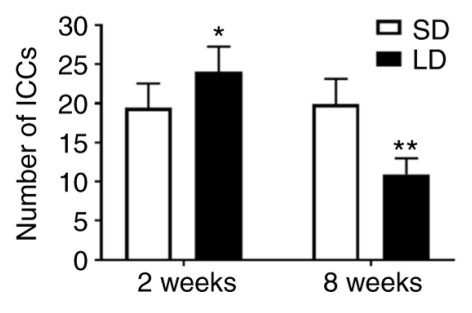

2 weeks
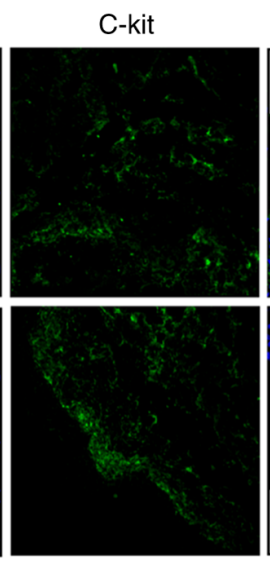
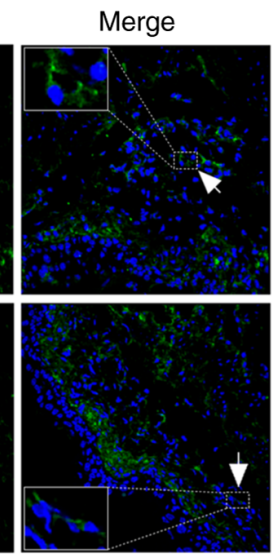

C

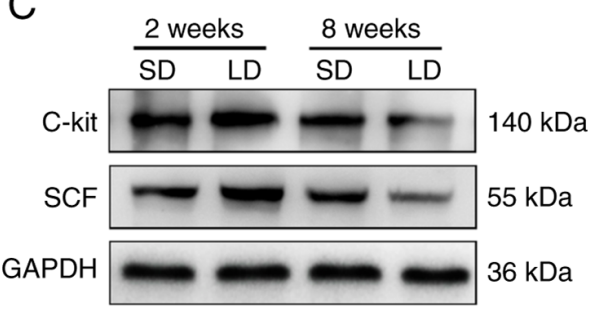

8 weeks

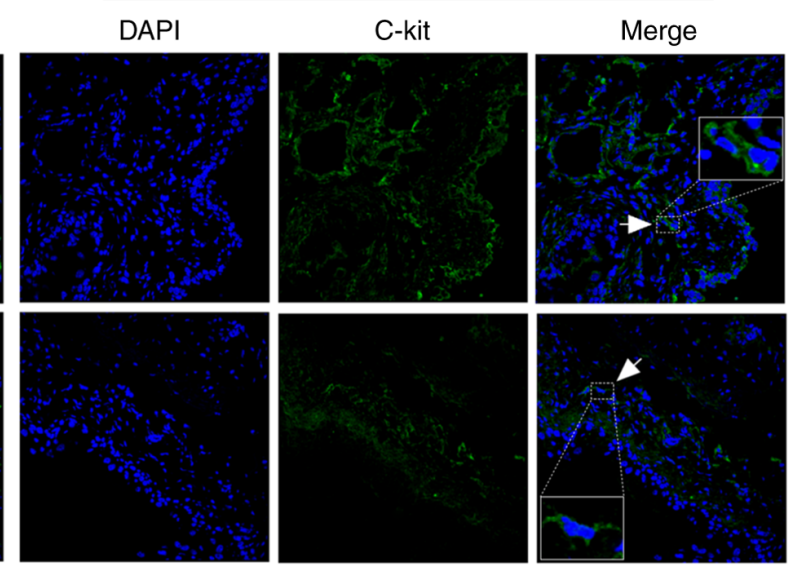

$\mathrm{D}$

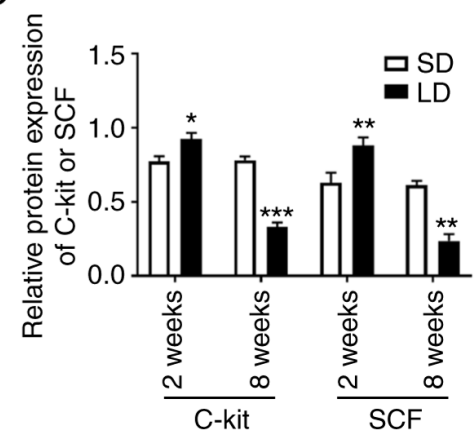

Figure 2. LD alters the number of ICCs and protein expression levels of C-kit and SCF in the gallbladders of guinea pigs. (A) Immunofluorescence staining of ICCs in the gallbladders of guinea pigs fed a SD or LD. ICCs were labelled using a primary antibody targeting C-kit (green), and nuclei were labelled using DAPI (blue). White arrowheads indicate C-kit-positive gallbladder ICCs, which are shown in the inset. Magnification, x200. (B) The mean number of ICCs in a field of view of guinea pig gallbladder. (C and D) Western blotting of C-kit and SCF protein expression levels in guinea pig gallbladders of the $\mathrm{SD}$ and LD groups. ${ }^{*} \mathrm{P}<0.05,{ }^{* *} \mathrm{P}<0.01$ and ${ }^{* * *} \mathrm{P}<0.001$ vs. $\mathrm{SD} / 2$ weeks or SD/8 weeks. SD, standard diet; LD, lithogenic diet; ICCs, interstitial cells of Cajal; $\mathrm{SCF}$, stem cell factor.

normal cell nuclei, clear cell contours and multiple branches. Compared with the SD/2 weeks group, the ultrastructure of gallbladder ICCs in guinea pigs of the LD/2 weeks group was not notably altered. However, in guinea pigs of the LD/8 weeks group, gallbladder ICCs exhibited more transparent cell nuclei, blurred cell outlines, enlarged mitochondria and several vacuoles in the cytoplasm. Notably, there were no notable branches observed in gallbladder ICCs in the LD/8 weeks guinea pigs (Fig. 3A). Furthermore, the protein expression levels of Cx43 in guinea pig gallbladders were increased by $\sim 37 \%$ in the $\mathrm{LD} / 2$ weeks group compared with the $\mathrm{SD} / 2$ weeks group, and was decreased by $\sim 66 \%$ in the LD/8 weeks group compared with that in the $\mathrm{SD} / 8$ weeks group (Fig. $3 \mathrm{~B}$ and $\mathrm{C}$ ).

CCK1R expression in gallbladder ICCs was significantly altered in guinea pigs fed a LD. The effects of LD on CCK1R expression in guinea pig gallbladders were determined. The serum levels of CCK8 in guinea pigs of the LD/2 weeks group were significantly increased compared with the SD/2 weeks group (SD/2 weeks vs. LD/2 weeks: $16.264 \pm 1.216 \mathrm{pg} / \mathrm{ml}$ vs. $18.934 \pm 0.822 \mathrm{pg} / \mathrm{ml}$ ). Compared with the SD/8 weeks group, the increase in the serum levels of CCK8 in guinea pigs fed a LD for 8 weeks were more significant (SD/8 weeks vs. LD/8 weeks: $16.168 \pm 1.065 \mathrm{pg} / \mathrm{ml}$ vs. $25.812 \pm 1.174 \mathrm{pg} / \mathrm{ml}$; Fig. 4A). Moreover, compared with the SD groups, the total protein expression levels of CCK1R in guinea pig gallbladders were significantly increased by $\sim 27 \%$ in the LD/2 weeks group, and was significantly decreased by $\sim 63 \%$ in the LD/8 weeks group (Fig. 4B and C). Finally, using double immunofluorescence staining, CCK1R (red fluorescence) was confirmed to be expressed in C-kit-positive (green fluorescence) ICCs in guinea pig gallbladders. Additionally, the red fluorescence intensity (indicative of CCK1R expression levels) in the gallbladder ICCs of guinea pigs in the LD/2 weeks group was moderately higher compared with the SD/2 weeks group; and was markedly lower in the LD/8 weeks group compared with the SD/8 weeks group (Fig. 4D).

\section{Discussion}

It was previously shown that hypercholesterolemia is an important risk factor of cholelithiasis $(18,19)$. Hypercholesterolemia can increase biliary cholesterol concentrations and consequently lead to cholesterol saturation in bile, which is also considered a pathogenic factor of cholelithiasis (20). In the present study, hypercholesterolemia was successfully induced, and elevated levels of T-CHOL in the bile from guinea pigs were observed when fed a LD for 2 or 8 weeks. Several studies have shown that a LD facilitates the formation of cholesterol gallstones in guinea pigs $(21,22)$. Hence, in the present study, 
A

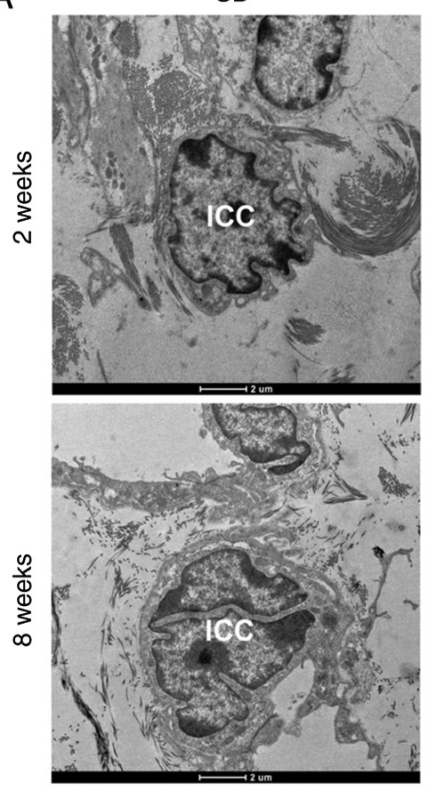

$$
\text { B }
$$

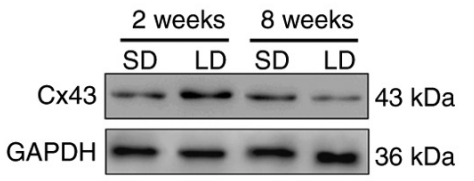

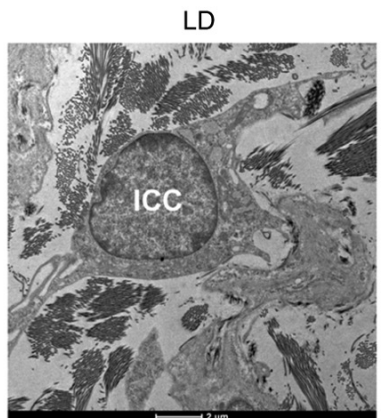
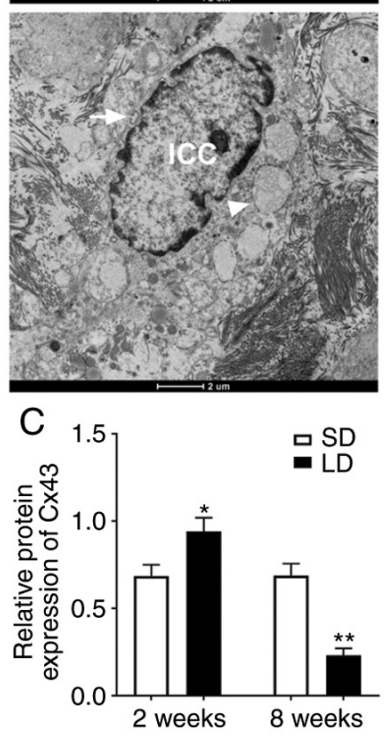

Figure 3. LD alters the ultrastructure of gallbladder ICCs in SD-fed guinea pigs after 8 weeks and the protein expression levels of $\mathrm{Cx} 43$ in guinea pig gallbladders. (A) Ultrastructure of gallbladder ICCs in the guinea pigs fed with SD or LD was visualized using a transmission electron microscope. The white arrowhead indicated enlarged mitochondria and the white triangle indicates the vacuole in the cytoplasm. Magnification, x10,000. Scale bar, $2 \mu \mathrm{m}$. (B and C) Western blotting of Cx43 expression in guinea pig gallbladders fed with SD or LD. ${ }^{*} \mathrm{P}<0.05,{ }^{* *} \mathrm{P}<0.01$ vs. SD/2 weeks or SD/8 weeks. SD, standard diet; LD, lithogenic diet; Cx43, connexin 43; ICCs, interstitial cells of Cajal.

LD-fed guinea pigs were used to investigate the possible mechanisms underlying gallbladder hypomotility in the development of cholesterol cholelithiasis.

ICCs are pacemaker cells that generate electrical slow waves in the gastrointestinal tract (23). ICCs have also been shown to serve a key regulatory role in controlling smooth muscle contractions in the urinary tract (24). In the gallbladder, ICCs are involved in the generation and propagation of spontaneous rhythmicity and the consequent tissue motility (9). A previous study found that gallbladder ICCs were redistributed from the muscle layer to the lamina propria during cholelithiasis (11). According to this result, in the present study, the changes in density of gallbladder ICCs located in the lamina propria were investigated. To the best of our knowledge, the present study is the first to show that a LD for 2 weeks significantly increases the density of gallbladder ICCs in guinea pigs. In addition, the density of gallbladder ICCs was significantly decreased in guinea pigs after being fed a LD for 8 weeks. This result was consistent with previous studies where the density of ICCs was found to be significantly reduced in patients with cholelithiasis (10) and in guinea pigs fed a high cholesterol diet for 4 or 8 weeks $(12,25)$. Based on the aforementioned results, it was hypothesized that guinea pigs may enhance their gallbladder emptying capacity via a compensatory increase in the density of ICCs during the early stages of high cholesterol stimulation, and their gallbladder motility is considerably impaired at the later stages of cholesterol cholelithiasis development due to decreases in the density of ICCs.

Subsequently, the mechanisms underlying the decrease in the density of gallbladder ICCs were investigated. The $\mathrm{SCF} / \mathrm{C}$-kit pathway is well established as a key regulator of the differentiation and proliferation of ICCs (26). The present study detected for the first time that the protein expression levels of SCF and C-kit in guinea pig gallbladders were significantly increased after feeding a LD for 2 weeks, indicating increased activation of the SCF/C-kit pathway. Furthermore, consistent with previous studies in which the mRNA and protein expression levels of SCF and C-kit were found to be significantly attenuated in the gallbladders of animal models fed a high cholesterol diet for 8 weeks $(12,27)$, the present study also demonstrated that activity of the SCF/C-kit pathway was significantly suppressed after being fed a LD for 8 weeks. Taken together, it was hypothesized that differential activity of the SCF/C-kit pathway is observed at various stages of cholesterol cholelithiasis development, and this may lead to the corresponding alterations in the density of gallbladder ICCs at each stage.

A previous study reported that feeding a high cholesterol diet for 8 weeks did not significantly result in ultrastructural changes in the gallbladder ICCs in mice (12). However, in the present study, although the ultrastructure of gallbladder ICCs in guinea pigs was not notably altered after feeding a LD for 2 weeks, they were markedly altered after 8 weeks. It was hypothesized that the differences in outcomes may be attributed to the different susceptibilities of various species to high cholesterol diets. It was reported that gallbladder ICCs can form a network with each other and close contact with smooth muscle cells and nerve endings through their multiple branches (9). Moreover, it was also shown that ICCs can propagate their own rhythmic excitation or neural signals to smooth muscle cells via gap junctions (28). In the present study, an important change that was observed in the ultrastructure of gallbladder ICCs at 8 weeks was the loss of their branched morphology. Additionally, the protein expression levels of the gap junction protein $\mathrm{Cx} 43$ in guinea pig gallbladders was significantly decreased after 8 weeks. Based on the aforementioned results, it was hypothesized that the capacity of ICCs for controlling gallbladder contraction via propagating rhythmic signals to smooth muscle cells may be impaired at the later stages of cholesterol cholelithiasis progression.

As a classical hormone, CCK can induce gallbladder contractions via direct action on CCK1R in gallbladder ICCs (15). In patients with cholesterol gallstones, induction of gallbladder contraction via exogenous CCK is impaired, and this phenomenon may be the result of reduced CCK1R expression and/or CCK binding ability to CCK1R in the gallbladder (29). Furthermore, a previous study reported that total CCK1R protein expression in gallbladders of mice fed with LD for 1 month was significantly reduced compared with the control mice (30). Consistently, the results of the present study also showed that CCK1R protein expression in the gallbladder, particularly in gallbladder ICCs, was significantly decreased 
A

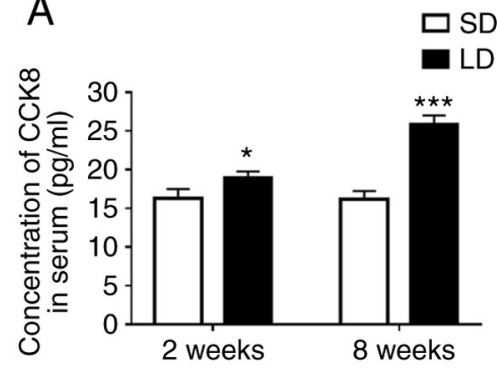

B

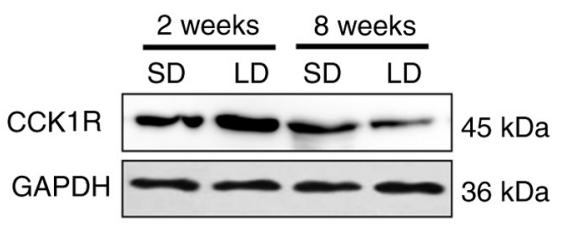

$\mathrm{C}$

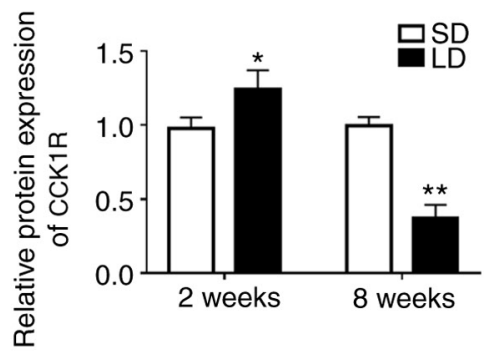

D

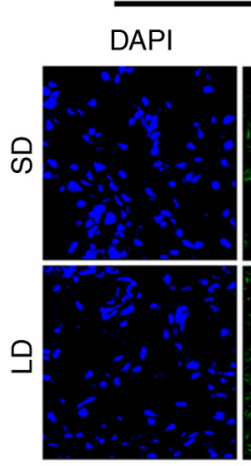

2 weeks

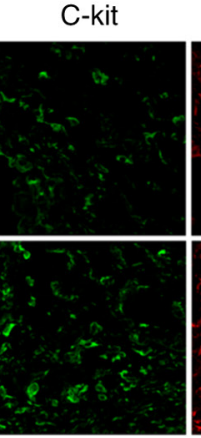

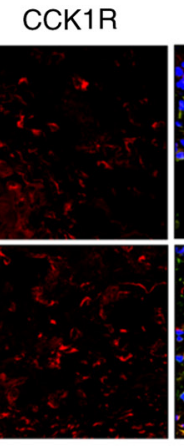

Merge

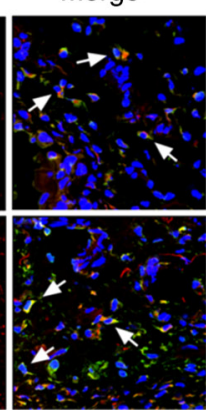

8 weeks

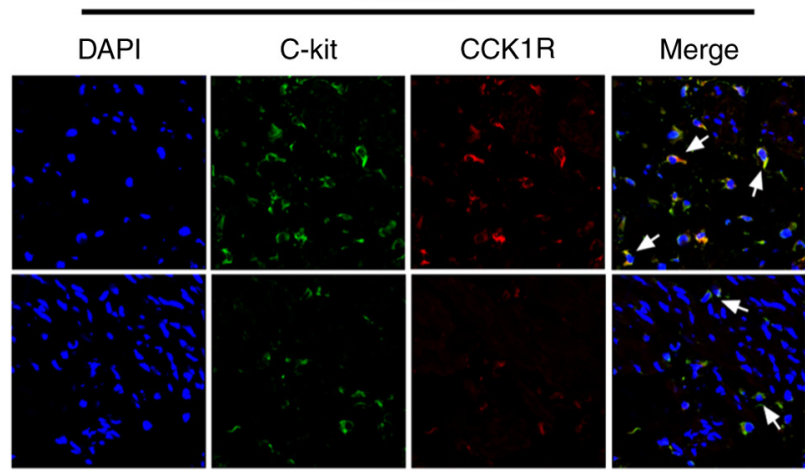

Figure 4. CCK1R expression in gallbladder ICCs is altered in guinea pigs fed a LD. (A) Serum CCK8 levels were measured after 2 and 8 weeks in guinea pigs fed a SD or LD. (B and C) Western blotting for CCK1R protein expression in guinea pig gallbladders fed a SD or LD. (D) Immunofluorescence labelling of CCK1R (red) expression in C-kit (green)-positive gallbladder ICCs of guinea pigs fed a SD or LD. Magnification, x400. Cell nuclei were labelled using DAPI (blue). White arrowheads indicate C-kit-positive gallbladder ICCs. ${ }^{*} \mathrm{P}<0.05,{ }^{* *} \mathrm{P}<0.01$ and ${ }^{* * * *} \mathrm{P}<0.001$ vs. SD/2 weeks or SD/8 weeks. SD, standard diet; LD, lithogenic diet; ICCs, interstitial cells of Cajal; CCK1R, cholecystokinin receptor type A.

after feeding with LD for 8 weeks. Although the serum CCK8 levels in guinea pigs were considerably increased after 8 weeks in the present study, it was hypothesized that gallbladder contraction was still damaged at this stage, primarily due to reduced CCK1R expression. In addition, the results of the present study also demonstrated for the first time that CCK1R protein expression in the gallbladder, particularly in the gallbladder ICCs was increased after feeding with LD for 2 weeks. Considering that the serum CCK8 levels in guinea pigs was also moderately increased after 2 weeks, it was hypothesized that the synergistic effect of increased serum CCK8 and CCK1R expression will enhance the emptying capacity of the gallbladder during the early stages of high cholesterol stimulation.

The present study preliminarily investigated changes in the density, ultrastructure and CCK1R expression of gallbladder ICCs during the development of cholesterol cholelithiasis. A limitation of the present study is that gallbladder contractility in guinea pigs was not assessed directly using functional experiments. Additionally, further studies are required to clarify the possible mechanisms underlying the changes in CCK1R expression, such as elucidation of the regulation of CCK1R expression by certain microRNAs, during the development of cholesterol cholelithiasis.

In conclusion, the present study demonstrated that the density, ultrastructure and CCK1R expression levels of gallbladder ICCs were differentially altered at various stages of cholesterol cholelithiasis progression. These results indicated that gallbladder ICCs may serve as a potential therapeutic target for cholesterol cholelithiasis.

\section{Acknowledgements}

Not applicable.

\section{Funding}

This study was funded by the Surface Project of Natural Science Foundation of Jiangsu Province (grant no. BK 20181491).

\section{Availability of data and materials}

The datasets used and/or analysed during the current study are available from the corresponding author on reasonable request.

\section{Authors' contributions}

LH and CD performed the experiments and analysed the data. LH wrote the manuscript. XS designed the experiments and revised the manuscript. $\mathrm{LH}, \mathrm{CD}$ and XS confirm the authenticity of all the raw data. All authors read and approved the final manuscript.

\section{Ethics approval and consent to participate}

All animal experiments were performed according to the National Institutes of Health Guide for the Care and Use of Laboratory Animals, and approved by the Institutional Animal Care and Use Committee of the First Affiliated Hospital of Nanjing Medical University (Nanjing, China). 


\section{Patient consent for publication}

Not applicable.

\section{Competing interests}

The authors declare that they have no competing interests.

\section{References}

1. Munoz LE, Boeltz S, Bilyy R, Schauer C, Mahajan A, Widulin N, Gruneboom A, Herrmann I, Boada E, Rauh M, et al: Neutrophi extracellular traps initiate gallstone formation. Immunity 51 443-450.e4, 2019.

2. Cai JS, Qiang S and Bao-Bing Y: Advances of recurrent risk factors and management of choledocholithiasis. Scand J Gastroenterol 52: 34-43, 2017.

3. Figueiredo JC, Haiman C, Porcel J, Buxbaum J, Stram D, Tambe N, Cozen W, Wilkens L, Le Marchand L and Setiawan VW: Sex and ethnic/racial-specific risk factors for gallbladder disease. BMC Gastroenterol 17: 153, 2017.

4. Chen CH, Lin CL and Kao CH: Association between Hashimoto's thyroiditis and cholelithiasis: A retrospective cohort study in Taiwan. BMJ Open 8: e020798, 2018.

5. Lammert F, Gurusamy K, Ko CW, Miquel JF, Mendez-Sanchez N, Portincasa P, van Erpecum KJ, van Laarhoven CJ and Wang DQ: Gallstones. Nat Rev Dis Primers 2: 16024, 2016.

6. Wang HH, Portincasa P, Liu M, Tso P, Samuelson LC and Wang DQ: Effect of gallbladder hypomotility on cholestero crystallization and growth in CCK-deficient mice. Biochim Biophys Acta 1801: 138-146, 2010.

7. Pasternak A, Szura M, Gil K and Matyja A: Interstitial cells of Cajal-systematic review. Folia Morphol 75: 281-286, 2016.

8. Radenkovic G, Radenkovic D and Velickov A: Development of interstitial cells of Cajal in the human digestive tract as the result of reciprocal induction of mesenchymal and neural crest cells. J Cell Mol Med 22: 778-785, 2018.

9. Chen L and Yu B: Telocytes and interstitial cells of Cajal in the biliary system. J Cell Mol Med 22: 3323-3329, 2018.

10. Pasternak A, Gil K, Matyja A, Gajda M, Sztefko K, Walocha JA, Kulig J and Thor P: Loss of gallbladder interstitial Cajal-like cells in patients with cholelithiasis. Neurogastroenterol Motil 25: e17-e24, 2013.

11. Spetana J, Lipinski A and Jelen M: Comparison of the ICC location in the gallbladder wall in patients with cholelithiasis and patients with non-calculous changes. Pol J Pathol 70: 205-209, 2019.

12. Fan Y, Wu S, Fu B, Weng C and Wang X: The role of interstitial Cajal-like cells in the formation of cholesterol stones in guinea pig gallbladder. Hepatol Int 9: 612-620, 2015.

13. Konno K, Takahashi-Iwanaga H, Uchigashima M, Miyasaka K, Funakoshi A, Watanabe $\mathrm{M}$ and Iwanaga T: Cellular and subcellular localization of cholecystokinin (CCK)-1 receptors in the pancreas, gallbladder, and stomach of mice. Histochem Cell Biol 143: 301-312, 2015.

14. Rathore RM, Angotzi AR, Jordal AE and Ronnestad I: Cholecystokinin receptors in Atlantic salmon: Molecular cloning, gene expression, and structural basis. Physiol Rep 1: $\mathrm{e} 00069,2013$.

15. Xu D, Yu BP, Luo HS and Chen LD: Control of gallbladder contractions by cholecystokinin through cholecystokinin-A receptors on gallbladder interstitial cells of Cajal. World J Gastroenterol 14: 2882-2887, 2008.
16. Tharp KM, Khalifeh-Soltani A, Park HM, Yurek DA, Falcon A, Wong L, Feng R, Atabai K and Stahl A: Prevention of gallbladder hypomotility via FATP2 inhibition protects from lithogenic diet-induced cholelithiasis. Am J Physiol Gastrointest Liver Physiol 310: G855-G864, 2016.

17. Liu M, Liu C, Chen H, Huang X, Zeng X, Zhou J and Mi S: Prevention of cholesterol gallstone disease by schaftoside in lithogenic diet-induced C57BL/6 mouse model. Eur J Pharmacol 815: 1-9, 2017.

18. Zheng Y, Xu M, Heianza Y, Ma W, Wang T, Sun D, Albert CM, Hu FB, Rexrode KM, Manson JE and Qi L: Gallstone disease and increased risk of mortality: Two large prospective studies in US men and women. J Gastroenterol Hepatol 33: 1925-1931, 2018.

19. Lee S, Kweon OK and Kim WH: Associations between serum leptin levels, hyperlipidemia, and cholelithiasis in dogs. PLoS One 12: e0187315, 2017.

20. Di Ciaula A, Garruti G, Fruhbeck G, De Angelis M, de Bari O, Wang DQ, Lammert F and Portincasa P: The role of diet in the pathogenesis of cholesterol gallstones. Curr Med Chem 26: 3620-3638, 2019

21. Wu X, Liang X, Du Y, Zhang Y, Yang M, Gong W, Liu B, Dong J, Zhang N and Zhang H: Prevention of gallstonesbyLidanGranule:Insightintounderlyingmechanismsusinga guinea pig model. Biomed Rep 5: 50-56, 2016.

22. Rong ZH, Chen HY, Wang XX, Wang ZY, Xian GZ, Ma BZ, Qin CK and Zhang ZH: Effects of sphincter of Oddi motility on the formation of cholesterol gallstones. World J Gastroenterol 22: 5540-5547, 2016.

23. Zheng H, Drumm BT, Zhu MH, Xie Y, O'Driscoll KE, Baker SA, Perrino BA, Koh SD and Sanders KM: $\mathrm{Na}^{+} / \mathrm{Ca}^{+}$exchange and pacemaker activity of interstitial cells of Cajal. Front Physiol 11: 230, 2020.

24. Sanders KM, Ward SM and Koh SD: Interstitial cells: Regulators of smooth muscle function. Physiol Rev 94: 859-907, 2014.

25. Huang ZP, Qiu H and Yu BP: Distribution changes of interstitial cells of Cajal during cholesterol gallstone formation in guinea pigs fed a high cholesterol diet. Int J Clin Exp Pathol 11: 1653-1659, 2018.

26. Chai Y, Huang Y, Tang H, Tu X, He J, Wang T, Zhang Q, Xiong F, Li D and Qiu Z: Role of stem cell growth factor/c-Kit in the pathogenesis of irritable bowel syndrome. Exp Ther Med 13: 1187-1193, 2017.

27. Fan Y, Wu S, Fu B, Yan X, Wang X and Zhang W: Decreased expression of stem cell factor mRNA and protein in the gallbladders of guinea pigs fed on high cholesterol diet. Int J Clin Exp Med 8: 6379-6383, 2015.

28. Wang L, Liang Y, Chen Q, Ahmed N, Wang F, Hu B and Yang P: Identification and distribution of the interstitial cells of Cajal in the abomasum of goats. Cell Transplant 27: 335-344, 2018.

29. Wang HH, Portincasa P and Wang DQ: Update on the molecular mechanisms underlying the effect of cholecystokinin and cholecystokinin-1 receptor on the formation of cholesterol gallstones. Curr Med Chem 26: 3407-3423, 2019.

30. Xu GQ, Xu CF, Chen HT, Liu S, Teng XD, Xu GY and Yu CH: Association of caveolin-3 and cholecystokinin A receptor with cholesterol gallstone disease in mice. World J Gastroenterol 20: 9513-9518, 2014.

This work is licensed under a Creative Commons Attribution-NonCommercial-NoDerivatives 4.0 International (CC BY-NC-ND 4.0) License. 